\title{
NUMERICAL CHARACTERIZATION OF A COMPOSITE BONDED WING-BOX
}

\author{
Stanley S. Smeltzer III ${ }^{1}$ and Andrew E. Lovejoy ${ }^{2}$ \\ NASA Langley Research Center, Hampton, VA 23681-2199 \\ Arunkumar Satyanarayana ${ }^{3}$ \\ ATK Space Division, Hampton, VA, 23681-2199
}

\begin{abstract}
The development of composite wing structures has focused on the use of mechanical fasteners to join heavily-loaded areas, while bonded joints have been used only for select locations. The focus of this paper is the examination of the adhesive layer in a generic bonded wing box that represents a "fastenerless" or unitized structure in order to characterize the general behavior and failure mechanisms. A global/local approach was applied to study the response of the adhesive layer using a global shell model and a local shell/solid model. The wing box was analyzed under load to represent a high-g up-bending condition such that the strains in the composite sandwich face sheets are comparable to an expected design allowable. The global/local analysis indicates that at these wing load levels the strains in the adhesive layer are well within the adhesive's elastic region, such that yielding would not be expected in the adhesive layer. The global/local methodology appears to be a promising approach to evaluate the structural integrity of the adhesively bonded structures.
\end{abstract}

\section{Introduction}

A number of National Aeronautics and Space Administration (NASA) and Department of Defense (DOD) programs have investigated the development of composite wing structures during the latter part of the twentieth century ${ }^{1-3}$. While each of these programs investigated the use of adhesively bonded joints for select locations, they all relied on mechanical fasteners to join the more heavily loaded regions. A more recent study conducted under the NASA Advanced Subsonic Technology Program investigated a unitized wing structure that combined two state-ofthe-art manufacturing processes, stitching and resin transfer molding (RTM), to reduce cost and weight ${ }^{4}$. Although the stitched-wing program eliminated fasteners and successfully correlated the numerical and experimental global response of the wing structure, the local response of joint and stiffener regions had higher than predicted strains and deformations. Additionally, the local inelastic response of the bonded/stitched joint regions was not investigated.

A goal of recent NASA aeronautics programs targeted for subsonic fixed wing aircraft is to reduce the empty mass-fraction of airframe components by as much as fifty percent. The development of mass-efficient, tailored joining technologies that eliminate fasteners is one strategy for achieving the fifty-percent mass-fraction goal. To aid in the development of "fastenerless" or unitized joint structures, NASA Langley conducted an in-depth, numerical study of a wing-box structure that corresponds to a generic commercial transport aircraft. The objectives of the present study were to characterize the general behavior and failure mechanisms of the wing-box as well as the local behavior of joint interfaces when subjected to a simulated high-g up-bending load. Additionally, a global/local analysis method was employed to evaluate the design for local regions of interest within the wing-box.

Local regions within a structure have been evaluated for at least the last half-century by taking the internal force and/or displacement fields in the larger structure and manually imposing them on the local feature or region of interest. A number of researchers in the early 1990's developed methods for performing global/local analyses of

\footnotetext{
${ }^{1}$ Structures Engineer, Structural Mechanics and Concepts Branch, Mail Stop 208, and AIAA Senior Member.

${ }^{2}$ Aerospace Engineer, Structural Mechanics and Concepts Branch, Mail Stop 190, and AIAA Senior Member.

${ }^{3}$ Aerospace Engineer, ATK Space Division, Mail Stop 190, and AIAA Member.
} 
structural components using the finite element analysis (FEA) method. Several methods require that a single model be created where a local model is embedded within a coarser, global model (refs. 5-7). Krishnamurthy and Raju ${ }^{5}$ use an independent refinement and integration procedure to couple independently-modeled global and local regions in a single analysis (local embedded in global). The local model is statically condensed to the boundary nodes, which are then related to the global model displacements using a spline interpolation function that satisfies the linear isotropic plate bending differential equations. Wang and Ransom ${ }^{6}$ use a coupled global/local analysis where local regions are embedded within a global model using interface technology. The interface technology allows for mismatched finite element models to be joined in a variationally-consistent manner, and reduces modeling complexity by eliminating transition meshing. Shell models are used and a single analysis is performed to obtain global and local results. Krueger and $\mathrm{O}^{\prime} \mathrm{Brien}^{7}$ use multipoint constraints to provide a kinematically-compatible interface between a local three-dimensional model and a global shell model. This is an embedded solid model within a shell model where a single analysis is performed. Other methods can be applied to global and local models that enable the user to use different analysis tools for each model (refs. 8-12). Whitcomb ${ }^{8}$ presented an iterative approach wherein a separate local model was effectively embedded within a global model of the overall structure. Given prescribed forces, displacement compatibility between the local and global models is assured by iteratively solving for the residual error between the two displacement fields and correcting the global displacements at the interface, which is then reapplied to the local model. This is done until the displacement residual is small, and then the responses in the local region can be examined. The method requires an under-relaxation factor if the local region is stiffer than the corresponding region in the global model to assist in convergence. $\mathrm{Li}$, et. al. ${ }^{9}$ apply interpolated displacements to a subcomponent "global" model that are obtained from a full global model. The results of the analysis using this subcomponent model are then evaluated against allowables obtained from local models and coupon tests of design details. Kilic, et. al. ${ }^{10}$ use a "global" element that includes the singular behavior at the junction of dissimilar materials with or without traction-free surfaces. It replaces a refined sub-model with this global element in the finite element model, which is the method developed by Barut et al. ${ }^{11}$. Young, et. al. ${ }^{12}$ use an iterative global/local analysis where a local model is effectively embedded within a global by applying displacements from global model to local model, applying residual loads to the global model, and iterating to convergence.

The present investigation was conducted to characterize the response of a bonded wing-box subjected to an upbending load and identify potential failure locations at the bonded interfaces using a global/local analysis procedure in a computationally efficient manner. The paper provides a description of the global/local analysis methodology applied to a generic bonded wing-box, and discusses the associated global and local finite element analysis results, and the failure modes predicted by the linear and nonlinear analyses for the simulated up-bending load.

\section{Global/Local Approach}

Global analysis provides a good means to evaluate the overall wing box response, however, to accurately evaluate the performance in the adhesive layer, a global/local approach is proposed. Many approaches have been used to perform global/local analysis as described in the introduction. The present approach is essentially that of Whitcomb ${ }^{8}$ with different convergence criteria. Convergence is obtained when a suitably normalized boundary work residual is less than the selected convergence criteria. This convergence criterion enforces equilibrium on the global/local boundary. A key advantage of the method is that the local model can have different boundary nodes and can even be analyzed using a different analysis code. This global/local procedure is depicted in Fig. 1 (where $\alpha$ represents the iteration counter), and is defined as follows:

The global/local procedure is depicted in Fig. 1, and is defined as follows:

1. An initial global analysis is performed and displacements are extracted along the global/local interface boundary to act as input boundary conditions for the local model. A mapping algorithm is required to transfer the displacements between the global and local models and is accomplished through a transformation matrix, [T], which is the identity matrix when the global and local boundary meshes are identical. Additionally, aerodynamic and other internally applied loads within the local region are applied to the local model.

2. The local model is analyzed and the boundary reaction loads (forces/moments) are computed at the global boundary points.

3. The boundary load residual is computed by subtracting the boundary reaction loads of the local model from the boundary reaction loads of the global model at the global/local interface.

4. The boundary load residual is calculated and the solution is checked for convergence. If convergence has been obtained, the process is complete. 


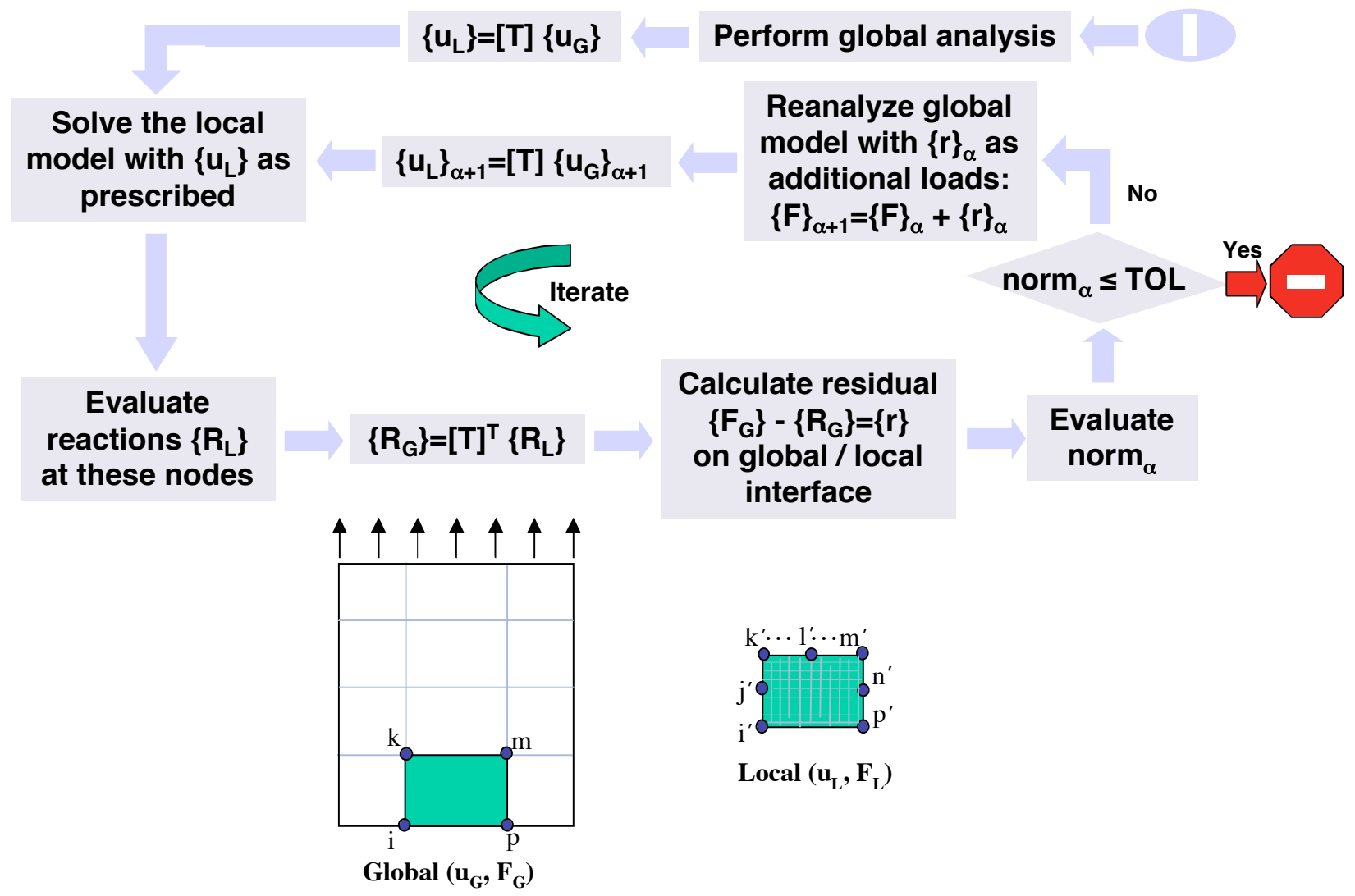

Figure 1. Global/local approach

5. If convergence has not been obtained, then the residual vector is introduced to the global model as an additional load set. That is, the total load set for the next iteration is the load set of the previous iteration plus the boundary residual load from the current iteration.

6. A global analysis is performed and displacements are extracted along the global/local interface boundary to act as input boundary conditions for the local model. Return to step 2) above.

Convergence was obtained by using a total boundary work residual in order to ensure that displacement and traction compatibility was maintained across the entire global/local interface. It represented the integrated work done at the interface between the global and local models and assessed the solution convergence in an overall energy sense. The total boundary work residual was normalized by the boundary work from the initial global analysis and compared to a small value (TOL), say 0.001 , to determine convergence. Mathematically this is given by Eq. 1, where $\mathrm{F}_{\mathrm{G}}$ and $\mathrm{u}_{\mathrm{G}}$ are the global model's global/local interface boundary force vector and displacement vector, respectively, and the subscript " 0 " indicates the initial global analysis (iteration zero).

$$
\text { norm }_{\alpha}=\frac{\sum_{\text {Boundary }}\left|\left(F_{G_{i}}\right)_{\alpha}\left[\left(u_{G_{i}}\right)_{\alpha}-\left(u_{G_{i}}\right)_{\alpha-1}\right]\right|}{\sum_{\text {Boundary }}\left|\left(F_{G_{i}}\right)_{0}\left(u_{G_{i}}\right)_{0}\right|} \leq T O L
$$

\section{Modeling Details}

The following section provides a detailed description of the global and local models used to characterize the wing-box response to an up-bending load and outlines the modeling approach. The generic wing box used in this paper has an outer mold line represented by the overall dimensions of 24 inches wide by 100 inches long by 6 inches deep (see Fig. 2). It comprises aluminum C-channel spars and composite sandwich panels having axially stiff composite inserts above the spar locations. A cross-section of the generic wing box is shown in Fig. 3. Bending strength for the box is provided by the spars and axially-stiffened inserts. The sandwich panels provide torsional stiffness and resist panel bending if the wing box were pressurized, such is the case with a wet wing containing fuel. 


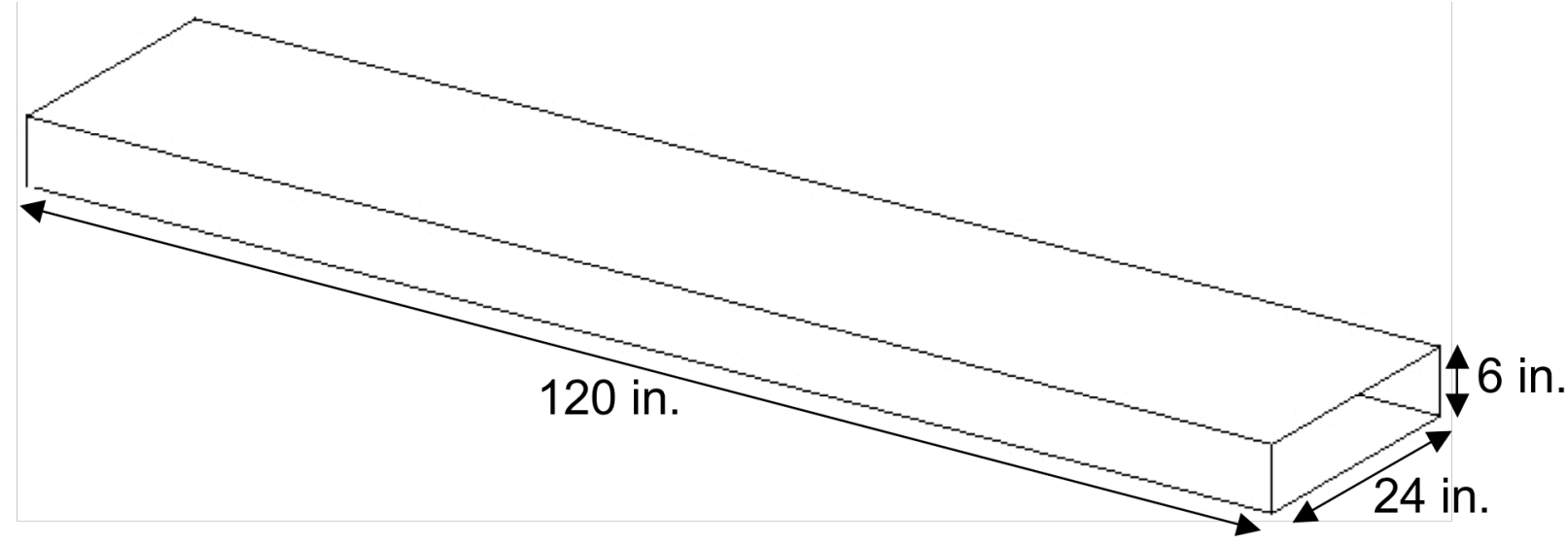

Figure 2. Outer mold line dimensions of generic wing box

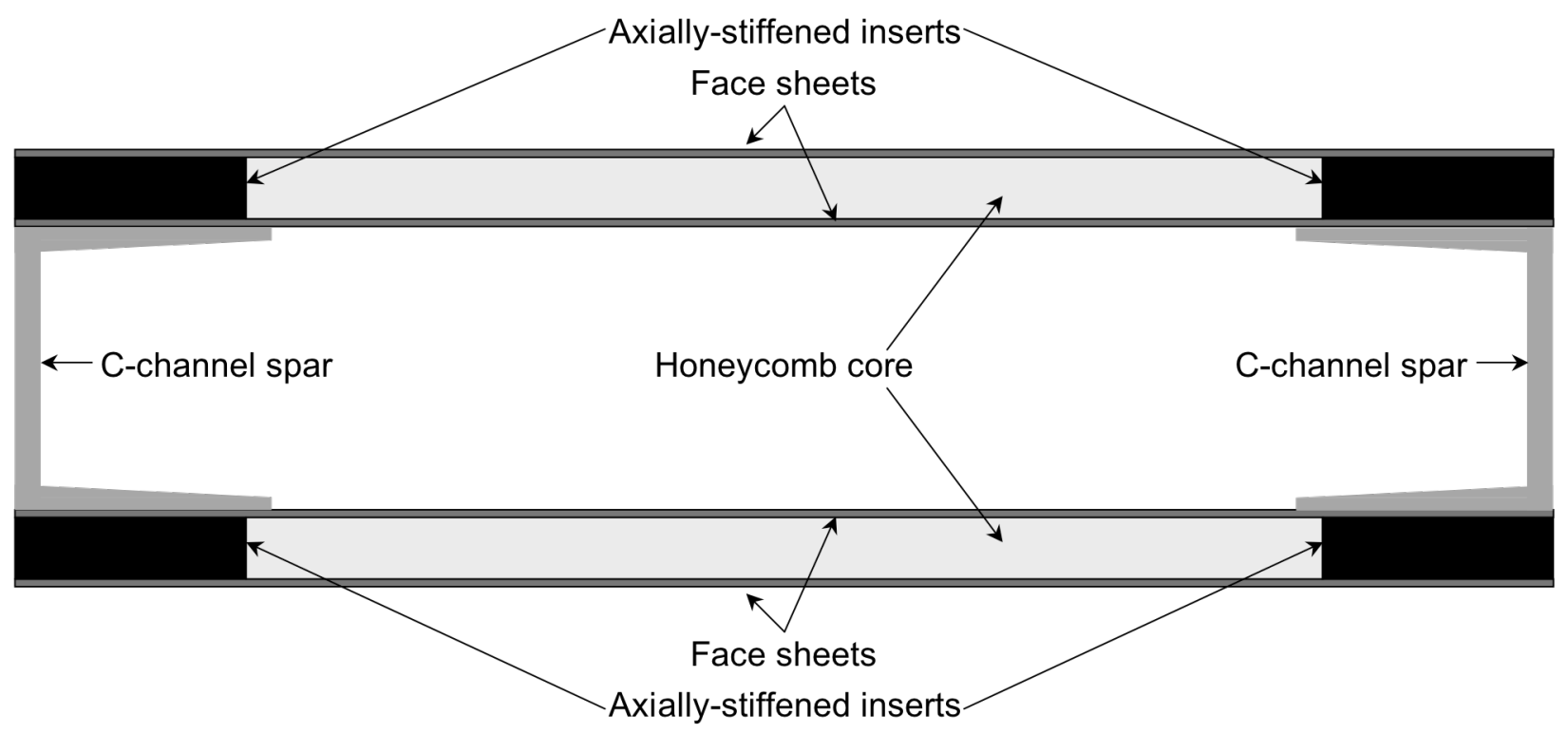

Figure 3. Cross-section sketch of generic wing box

The aluminum spars were assumed to be fabricated from extruded 6061 aluminum alloy, with properties from MILHDBK- $5^{13}$ Table 3.6.2.0(g), as given in Table 1. The sandwich face sheets and axially-stiffened inserts are fabricated from T800/3900-2, with the lamina properties shown in Table 1 as obtained from Knight ${ }^{14}$. The core of the sandwich panels is Hexcel aluminum honeycomb of type 1/4-5052-.003 with properties obtained from the manufacturer's literature ${ }^{15}$ and shown in Table 1.

The sandwich cover panel design consists of half-inch thick honeycomb core with three-ply cloth face sheets. Each face sheet was specified to be 0.025 inches thick, and was approximated using a six-ply laminate having uniaxial lamina with a stacking sequence of [45/-45/0/90/-45/45], and with the 90-degree ply closest to the core. The axially-stiffened inserts shown in Fig. 3 are two inches wide and half-inch thick to match the core thickness. To represent a laminate having percentages of [0/ $\pm 45 / 90]$ degree plies of [70/18/12], the axially-stiffened inserts were approximated by a 68-ply laminate with a stacking sequence of $\left[\left[0_{2} / 45 /-45 / 0_{3} / 90 / 0_{3} /-45 / 0_{3} / 90 / 0\right]_{\mathrm{s}}\right]_{\mathrm{s}}$. The aluminum $\mathrm{C}$-channels were assumed to be 4.9 inches in height with 2.25 -inch flanges. The web is 0.2 -inches thick, and the flanges taper from 0.15 to 0.042 -inches thick. The face sheets, core and axially-stiffened inserts were considered to be co-cured, then adhesively bonded to the spars. It is the adhesive layer between the inner face sheet and the Cchannel flange, attaching the sandwich panels to the spars, that is investigated in this paper.

\section{A. Global}

A global finite element model of the wing box was generated, and then linear analysis was performed using MSC/NASTRANTM. The model, shown in Fig. 4, was constructed using CQUAD4 shell elements located at the 
wing box outer mold line (outer surface of the sandwich cover panels, and outer surface of the C-channel spar webs). Appropriate offsets were applied to locate the shell element reference surfaces. The stiffness added by the spar web elements that are located through the sandwich panel thickness, closing the gap between the spar flanges and the cover panel OML, was considered to be negligible in the global model compared to the stiffness of the composite inserts, which provide the majority of the box bending stiffness. In an effort to approximate a high-g up-bending condition, the wing box was analyzed as if it were being tested in a three-point bend test. The resulting boundary conditions are pinned at the mid-height of the spars at each end, with a total load of 37.6 kips being applied at spar mid-height at the box centerline as shown in Fig 4. A load of 37.6 kips was chosen so that the maximum face sheet strain would be near to but not
Table 1. Material properties and strength data

\begin{tabular}{lll}
\hline \hline Properties & \multicolumn{1}{c}{ T800/3900-2 } & \multicolumn{1}{c}{ Description } \\
\hline $\mathrm{E}_{11}$ & $23.2\left(\times 10^{6} \mathrm{psi}\right)$ & Young's modulus, fiber dir. \\
$\mathrm{E}_{22}$ & $1.3\left(\times 10^{6} \mathrm{psi}\right)$ & Young modulus, transverse dir. \\
$\mathrm{G}_{12}, \mathrm{G}_{13}$ & $0.9\left(\times 10^{6} \mathrm{psi}\right)$ & $\begin{array}{l}\text { In-plane shear modulus } \\
\text { Poission's Ratio }\end{array}$ \\
$v_{12}$ & 0.28 & \multicolumn{1}{c}{ Description } \\
\hline \hline Properties & \multicolumn{1}{c}{ EA3980 } & \multicolumn{1}{c}{ Description } \\
\hline $\mathrm{E}$ & $0.234\left(\times 10^{6} \mathrm{psi}\right)$ & Young's modulus \\
$v_{12}$ & 0.3 & Poission's Ratio \\
$\boldsymbol{\varepsilon}$ & $45,000(\mu \varepsilon)$ & Yield strain \\
\hline \hline Properties & \multicolumn{1}{c}{ Aluminum } & \\
\hline $\mathrm{E}$ & $9.9\left(\times 10^{6} \mathrm{psi}\right)$ & Young's modulus \\
$v_{12}$ & 0.33 & Poission's Ratio \\
\hline \hline Properties & Al. H/C Core & \\
\hline $\mathrm{E}_{11}, \mathrm{E}_{22}$ & $0.001\left(\times 10^{6} \mathrm{psi}\right)$ & Young's modulus, in-plane \\
$\mathrm{E}_{33}$ & $0.235\left(\times 10^{6} \mathrm{psi}\right)$ & Young's modulus, transverse dir. \\
$v_{12}$ & 0.3 & Poission's Ratio \\
$\mathrm{G}_{13}$ & $0.096\left(\times 10^{6} \mathrm{psi}\right)$ & Transverse shear modulus \\
$\mathrm{G}_{23}$ & $0.0405\left(\mathrm{x} 10^{6} \mathrm{psi}\right)$ & Transverse shear modulus \\
\hline \hline
\end{tabular}

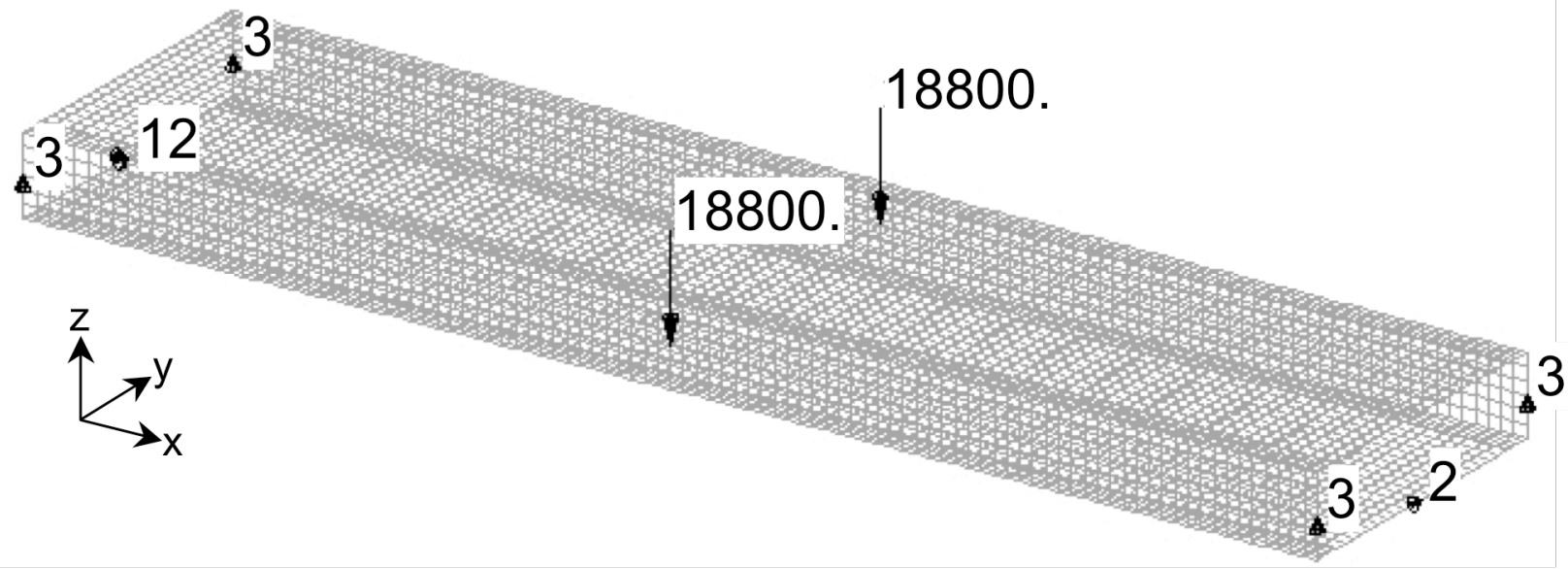

Figure 4. FEM and boundary conditions for generic wing box

exceed $5000 \mu \varepsilon$, which is a typical limiting design strain for this type of structure. This allows evaluation of the adhesive layer in a structure that is loaded to usual operating load levels. The additional boundary conditions shown in the figure, located on the lower cover free edges, are applied to remove the remaining rigid body motions and are applied at these locations for convenience.

\section{B. Local}

Analysis of complex structural parts that have many components of varying sizes and shapes require high fidelity finite element models to understand their behavior under complex loading conditions. More often, high fidelity finite element models consist of 3D finite elements whose sizes are small enough to accurately capture the stress and strain fields. However, such a modeling approach becomes computationally expensive when it is applied to the entire structure or part. Therefore, the present paper demonstrates a computationally efficient modeling approach using a combination of 2D shell and 3D solid elements to analyze a generic bonded wing box structure. The local model shown in Fig. 5(a) is a section of the full model and consists of 3D solid elements and 2D shell elements. The shell element region shown in the figure surround the solid element region, thereby making it easier to apply the translational and rotational displacement boundary conditions from the global $2 \mathrm{D}$ shell model to the 
local model. In the present study, the global and the local models have different mesh sizes, therefore, a linear interpolation function was used to map displacements and rotations from the global shell model to the local model. Within the local model, transition from shell to elements to solid elements was accomplished using the shell-to-solid coupling algorithm in the ABAQUS finite element code ${ }^{16}$ that was used for the local nonlinear analysis. Surface-based shell-to-solid coupling allows transition from shell element to solid element modeling using internally-defined distributing coupling constraints to couple the motion of a line of nodes along the edge of a shell model to the motion of a set of nodes on a solid surface ${ }^{16}$. Each internal constraint distributes the forces and moments acting at its shell (independent) node as a forces acting on the related set of solid (dependent) coupling surface nodes, thereby creating selfequilibrium between the two sets of nodes ${ }^{16}$.

The finite element model of the local region which shown in Fig. 5(b), consists of 52847 nodes, 6076 2D shell elements and 38750 3D solid elements. The 2D portion is modeled is using 4node (S4) quadrilateral elements. In the 3D portion, the C-channel, adhesive layer and honeycomb core were modeled using 8-node solid continuum (C3D8) elements, whereas the face sheets and the axially-stiffened inserts are modeled using 8-node shell continuum (SC8R) elements.

The C-channel and the honeycomb core are made up of aluminum whereas the face sheets and axially-stiffened inserts utilize laminates of T800/3900-2 material. An EA9380 adhesive material ${ }^{17}$ is used in bonding the skin (assembly of lower face sheet, axially-stiffened inserts, top face sheet and core) to the C-channel. The properties of the materials used in the local model analysis are provided in Table 1. The stacking sequences of face sheets and axially-stiffened inserts previously defined are repeated in Table 2. In this paper only failure within the adhesive is considered, not failure at the interface between the adhesive and adherents. Additionally, only the interior of the box is examined and the effects at the box ends are ignored.

Table 2. Stacking sequences of face sheets and axially stiff region

\begin{tabular}{lll}
\hline \hline \multicolumn{1}{c}{ ID } & \multicolumn{1}{c}{ Laminate Stacking Sequence } & Thickness (in.) \\
\hline Face sheets (top \& bottom) & {$[45 /-45 / 0 / 90 /-45 / 45]$} & 0.025 \\
Axially stiffened region & {$\left[0_{2} / 45 /-45 / 0_{3} / 90 / 0_{3} /-45 / 0_{3} / 90 / 0_{2} / 90 / 0_{3} /-45 / 0_{3} / 90 / 0_{3} /-45 / 45 / 0_{2}\right]_{\mathrm{s}}$} & 0.5 \\
\hline \hline
\end{tabular}

\section{Results and Discussion}

\section{A. Global}

The deformed shape of the wing box under the prescribed loading condition is shown in Fig. 6, where the deformations are magnified by a factor of 10 . The maximum vertical deflection at the spar/cover panel interface was seen to be -1.187 inches at the center of the box. Maximum span-wise strain in the cover panels was seen at the center of the wing box and has a value of $4500 \mu \varepsilon$ (tension and compression), which is close to but does not exceed the $5000 \mu \varepsilon$, as was desired. A local region at the center of the box is identified as shown in Fig. 7. The local model represents a region that is located at the center of the box, is bounded by the spar web centerline and the compression-side sandwich panel centerline, consists of 6 interface edges, and is shown in the figure as the red shaded area. The dimensions of this local region are 3 inches high by 12 inches wide by 30 inches long. For this 


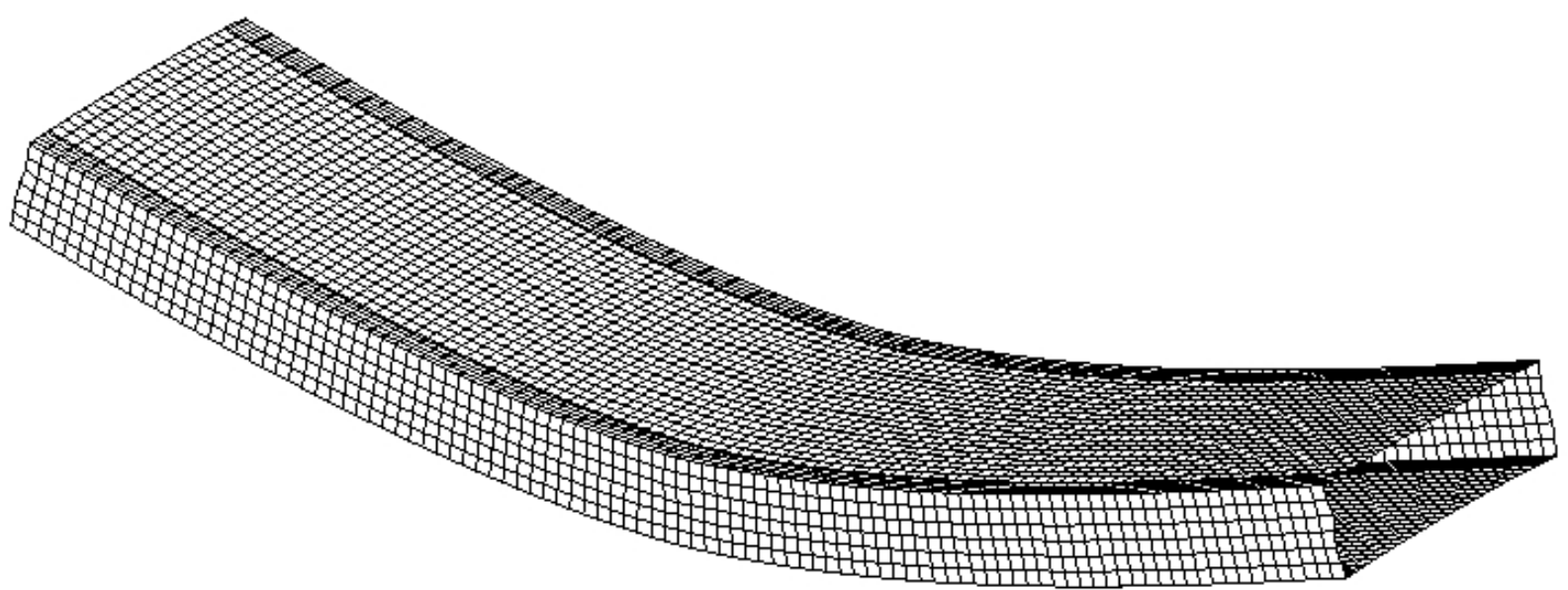

Figure 6. Global deformation (magnification 10x)

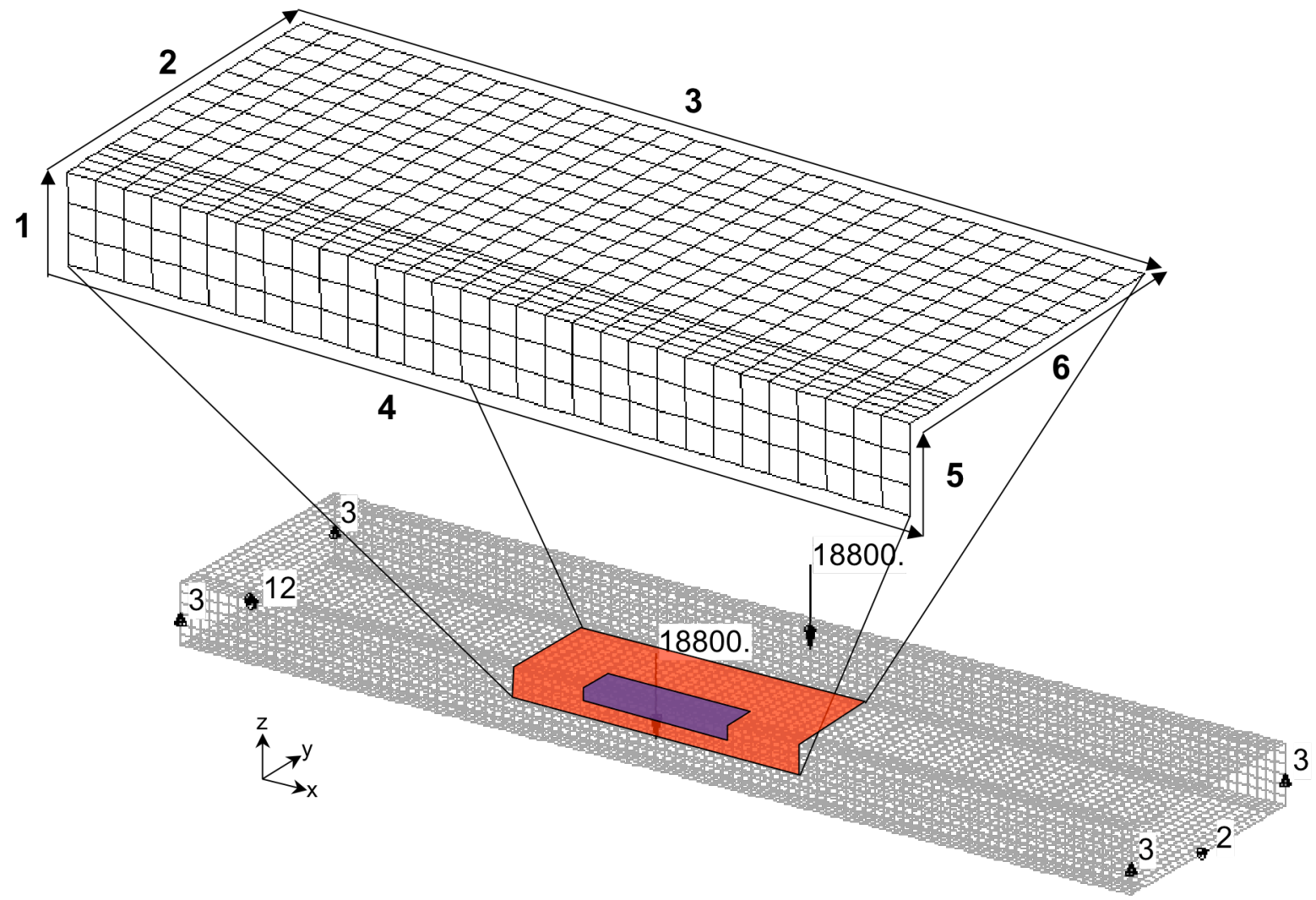

\section{Figure 7. Local region definitions}

simple wing box configuration and loading, it was found that no iterations were required, indicating that the relative stiffness of the local model and the local region of the global model are very close. A subregion, identified by the blue shading in Fig. 7 represents the solid portion of the detailed local model, whose size is shown in Fig. 5(a). The z-direction displacement contours for this subregion of the global model are shown in Fig. 8, and compare very favorably with the local detailed solid model (within about $1 \%$ ).

\section{B. Local}

Analysis results for the solid portion of the local model, including bending deformation and strains in the top face sheet, middle of the axially-stiffened insert and the adhesive layer, are presented in Figs. 9-15. While nonlinear analysis was used, it was seen that the response of the local model was linear, and the observed strains were well

American Institute of Aeronautics and Astronautics 
within the linear response regime. The out-of-plane deformation $\left(\mathrm{U}_{3}\right)$ of the solid model portion of the wing box obtained from the local 3D analysis is presented in Fig. 9. Comparison of the deformation contours shown in this figure with those shown in Fig 8 for the global analysis agree within about $0.5 \%$, thereby reinforcing the idea that a global-local approach can be used to accurately model and analyze large, complex structures with high fidelity. This result also suggests that the coupling constraints at the shell-to-solid interfaces accurately distribute the displacements and forces across the boundary. However, the results at the shell-solid interfaces should generally be ignored as the interfaces represent an internal boundary condition.

The span-wise wing box strains in the top face sheet are shown in Fig. 10 The maximum and minimum principal strains in the axially-stiffened insert are shown in Figs. 11 and 12, respectively. The values shown in these figures indicate that the strains are well below the allowable strain level, even at load levels near the maximum that a wing is expected to experience in real flight conditions.

The maximum and minimum principal strains in the adhesive layer of the local model, which

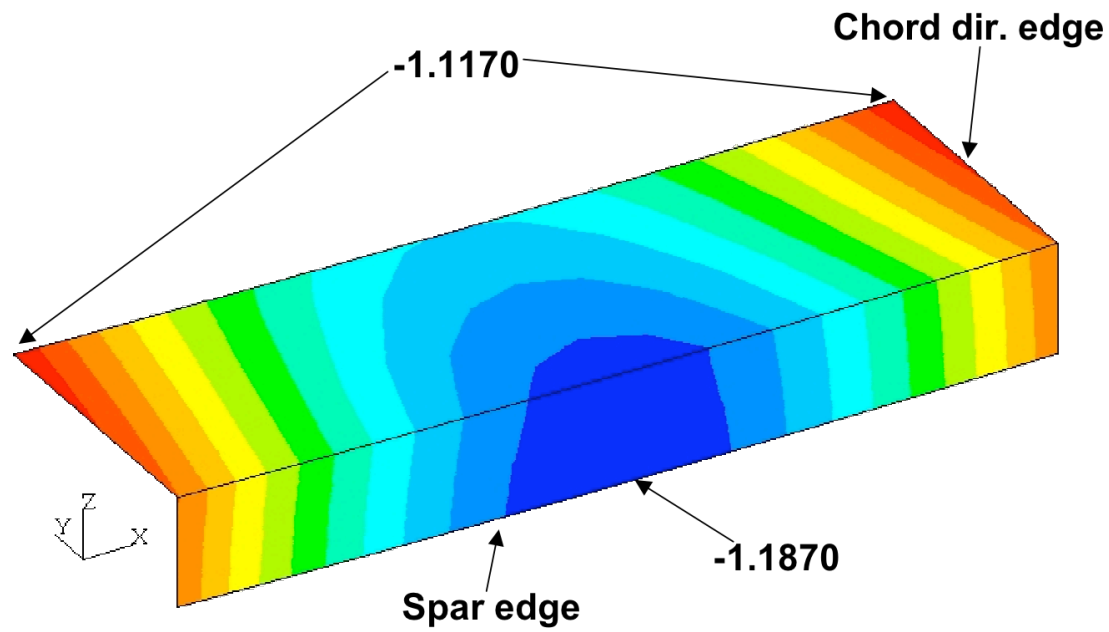

Figure 8. Z-direction deformation contours for global model region corresponding to local solid model region

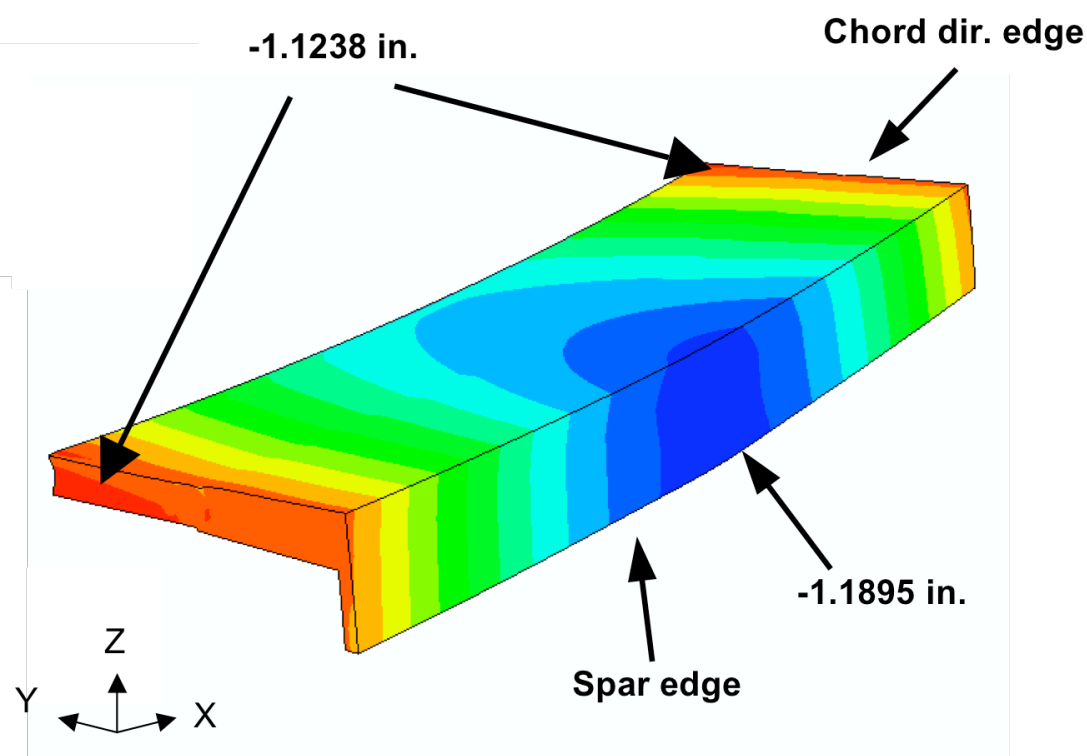

Figure 9. Deformation plot $\left(\mathrm{U}_{3}\right)$ of the local model

bonds the inner face sheet to the C-channel, are shown in Figs. 13 and 14, respectively. It should be noted that the distribution shown is a function of the applied point load condition and does not necessarily represent the response of the box under realistic flight load conditions. A maximum strain of about $9500 \mu \varepsilon$ is reached along the edge, and strain levels drastically reduce to about $2800 \mu \varepsilon$ away from the edge. The maximum strain value of about $9500 \mu \varepsilon$ is well within the allowable strain value of around $45000 \mu \varepsilon$ for the EA9380 adhesive. While the maximum principal strain plot indicates the point or region of high strain, it does not reveal the specific component(s) of the strain with the highest contribution. The axial, transverse, normal and shear strains are plotted for the adhesive in Fig. 15. It is seen in this figure that the strain components are all well below the values required to cause any failure in the adhesive layer based on a single point strain evaluation.

\section{Concluding Remarks}

A global/local approach was successfully applied to study the response of the adhesive layer in a generic wing box. The wing box was analyzed under load to approximate a high-g up-bending condition such that the strains in the composite sandwich face sheets are comparable to a reasonable design allowable. Results from this analysis 
indicate that no yielding is expected to develop within the adhesive layer. Further study is required to examine the response of the adhesive within more complex geometries and in the presence of defects such as a delamination.

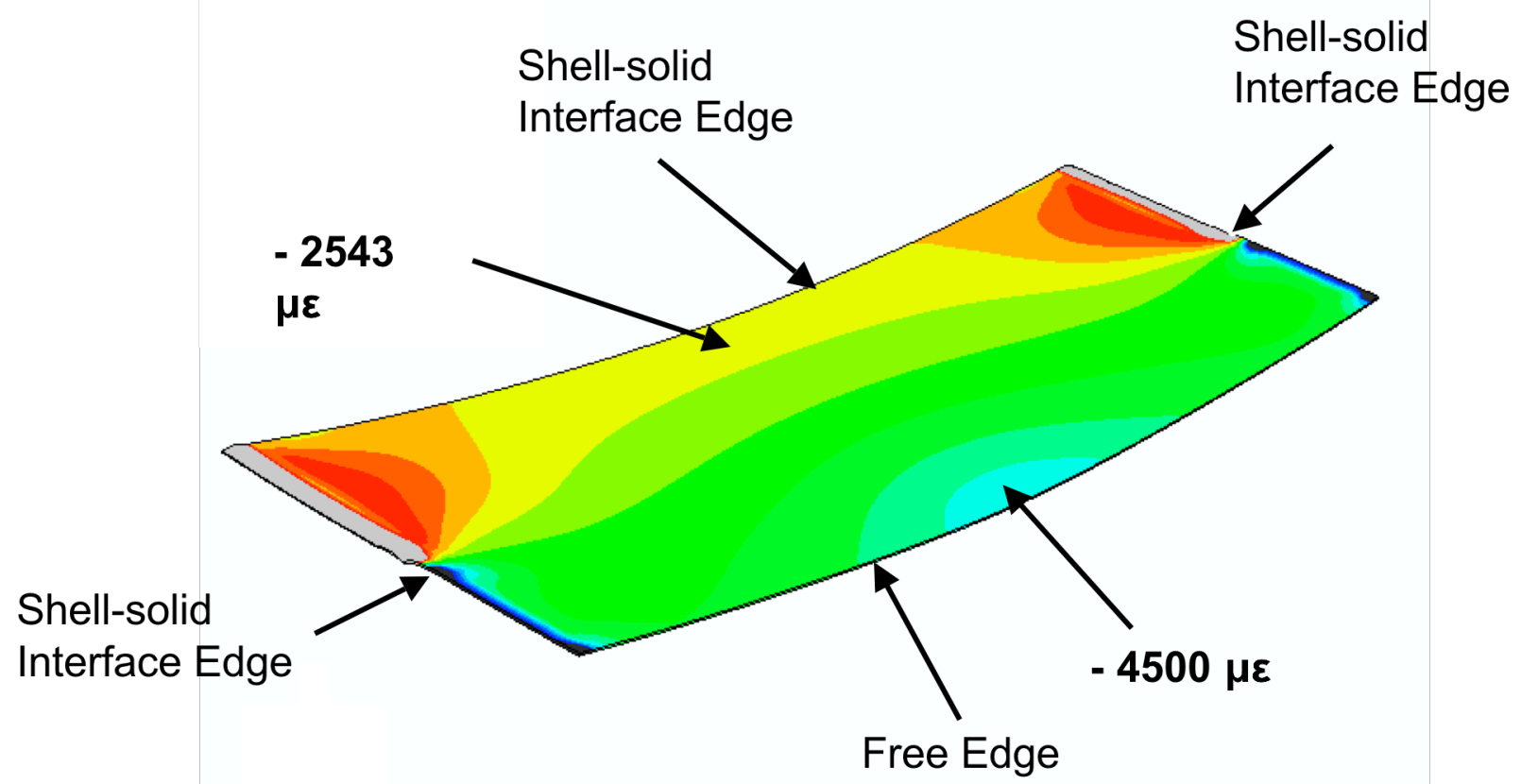

Figure 10. Span-wise strain in top face sheet

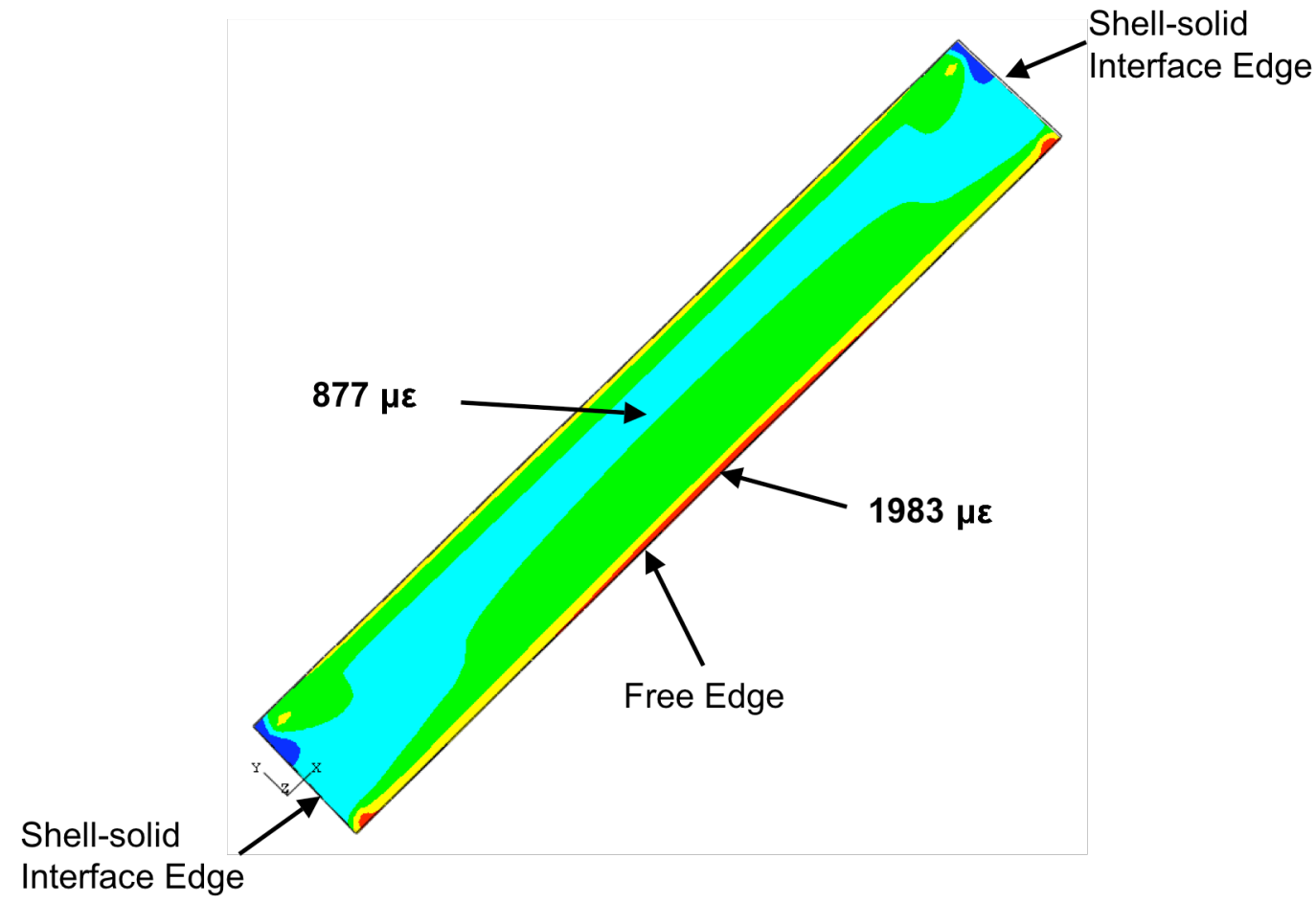

Figure 11. Maximum principal strain in axially stiffened region 


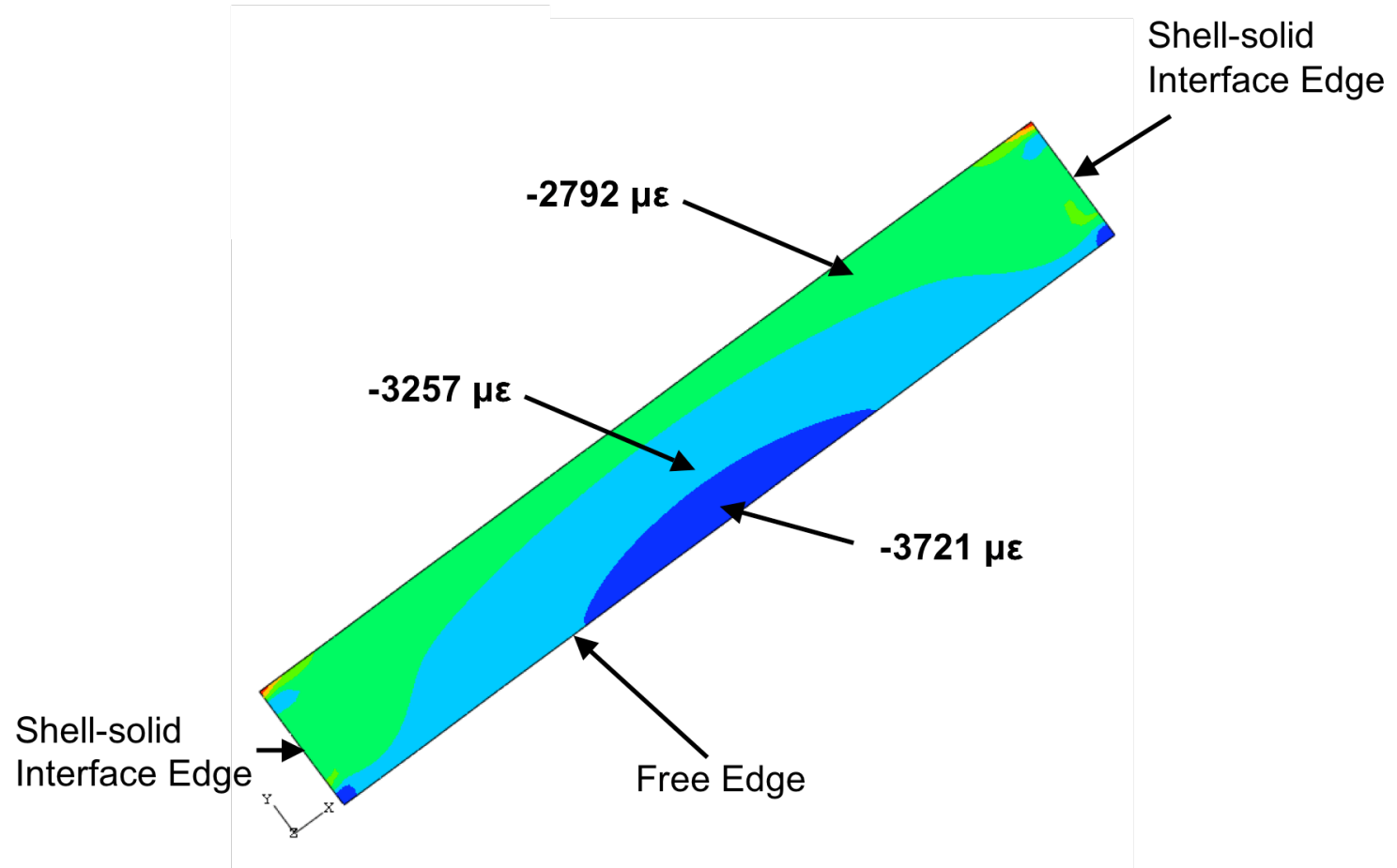

Figure 12. Minimum principal strain in axially stiffened region

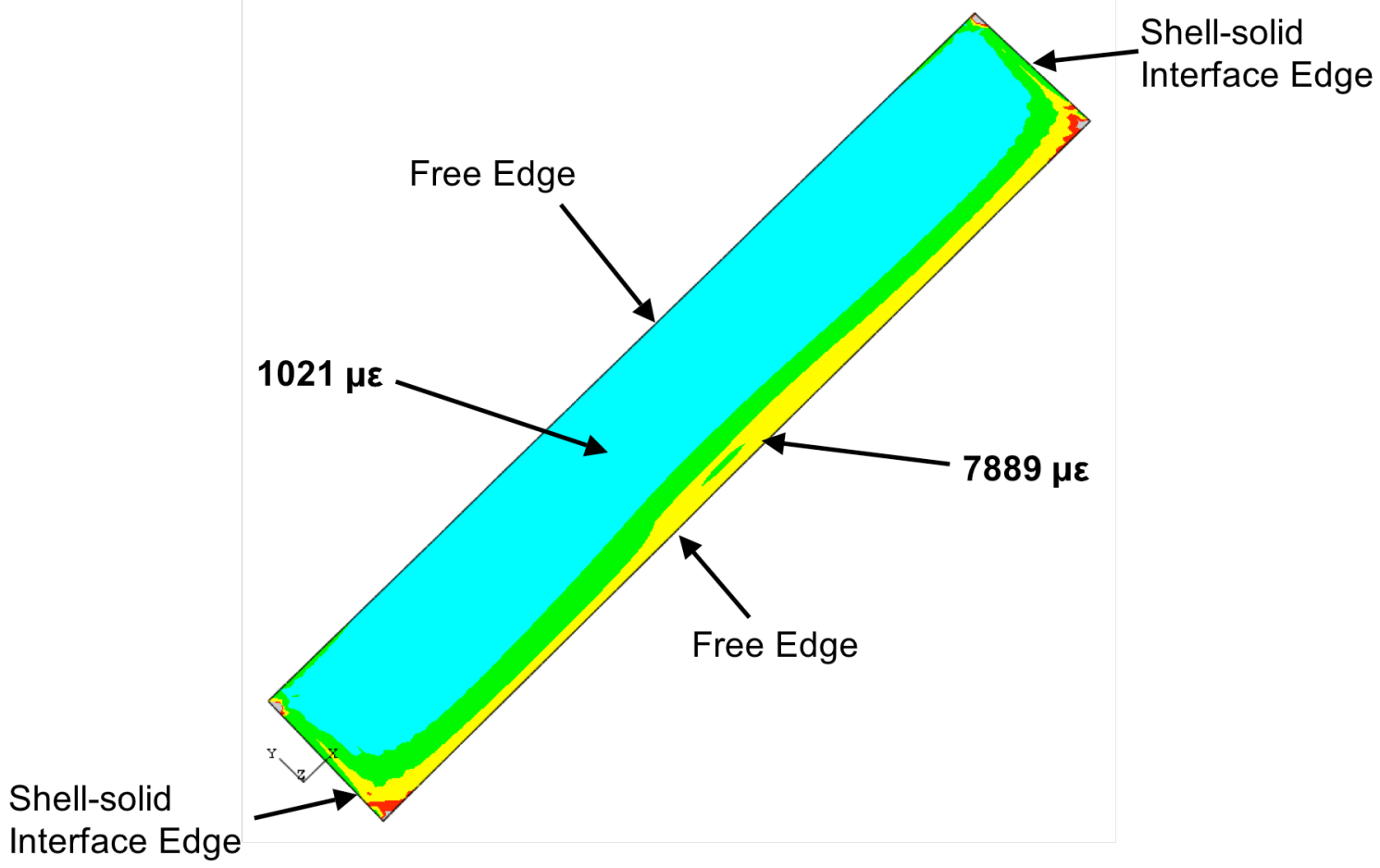

Figure 13. Maximum principal strain in the adhesive layer 


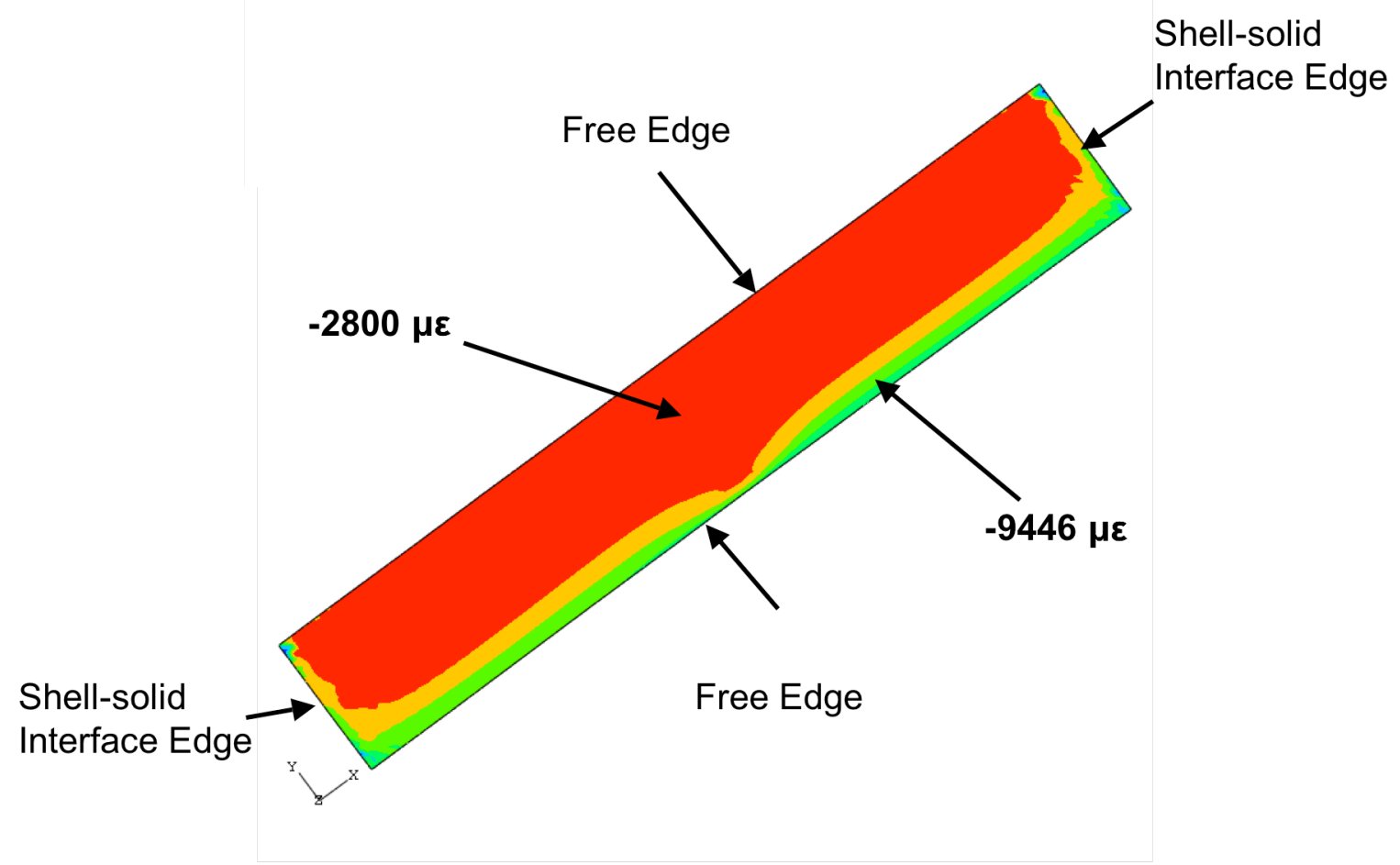

Figure 14. Minimum principal strain in the adhesive layer

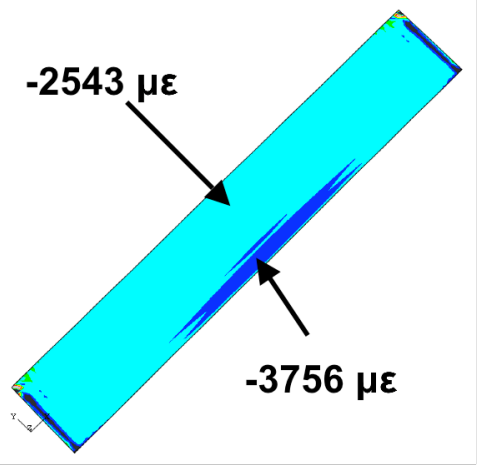

Strain in axial direction $(\mathrm{x})$

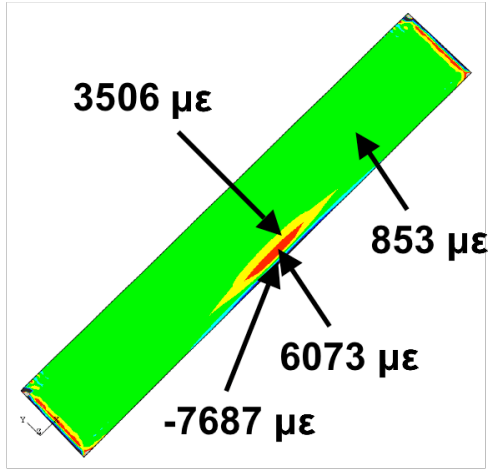

Strain in Normal direction (z)

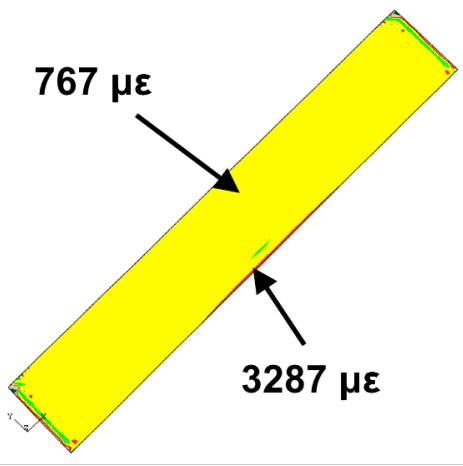

Strain in transverse direction (y)

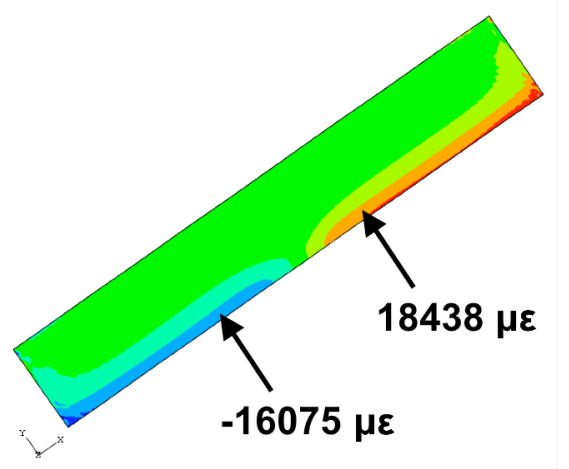

Strain in shear direction (xz)

Figure 15. Component strains in the adhesive layer 


\section{References}

${ }^{1}$ Hadcock, R.N. et al., “Advanced Composite Wing Structures,” AFML-TR-70-231, Volume I, December 1970.

2 Thrall, E.W., "Chapter 1 - The primary adhesively bonded structure technology (PABST) program," Adhesion 4 edited by K.W. Allen, Applied Science Publishers, London, 1980, pp. 1-54.

${ }^{3}$ Hawley, A., "Preliminary Design of a Transport Aircraft Composite Wing," Proceedings of the Fifth NASA/DoD Advanced Composites Technology Conference, NASA CP-3294, Vol. 1, Pt. 2, 1994, pp. 717-772.

${ }^{4}$ Jegley, D.C., Bush, H.G., and Lovejoy, A.E., "Structural Response and Failure of a Full-Scale Stitched Graphite Epoxy Wing," J. of Aircraft, Vol. 40, No. 6, 2003, pp. 1192-1199.

${ }^{5}$ Krishnamurthy, T., and Raju, I.S., "An Independent Refinement and Integration Procedure in Multiregion Finite Element Analysis," AIAA-92-2290.

${ }^{6}$ Wang, J. T. and Ransom, J. B., "Application of Interface Technology in Nonlinear Analysis of a Stitched/RFI Composite Wing Stub Box," AIAA-97-1190.

${ }^{7}$ Krueger, R., and O'Brien, T. K., "A Shell/3D Modeling Technique for the Analysis of Delaminated Composite Laminates," Composites Part A: Applied Science and Manufacturing, Vol. 32, No. 1, 2001, pp. 25-44.

${ }^{8}$ Whitcomb, J. D., "Iterative Global/local Finite Element Analysis," Computers \& Structures, Vol. 40, No. 4, 1991, pp. 10271031.

${ }^{9}$ Li, J., Davila, C. G., and Chen, T.-K., "High Fidelity Failure Analysis for a Composite Fuselage Section," American Helicopter Society 57th Annual Forum, Washington, DC, May 9-11, 2001.

${ }^{10}$ Kilic, B., Madenci, E., and Ambur, D. R., "Global-local Finite Element Analysis of Bonded Single-lap Joints," AIAA 20041559.

${ }^{11}$ Barut, A., Guven, I., and Madenci, E., "Analysis of Singular Stress Fields at Junctions of Multiple Dissimilar Materials Under Mechanical and Thermal Loading," International Journal of Solids \& Structures, Vol. 38, 2001, pp. $9077-9109$.

${ }^{12}$ Young, R. D., Lovejoy, A. E., Hilburger, M. W., and Moore, D. F., "Structural Analysis for the American Airlines Flight 587 Accident Investigation - Global Analysis," AIAA 2005-2254, 46th AIAA/ASME/ASCE/AHS/ASC Structures, Structural Dynamics \& Materials Conference, 18 - 21 April 2005, Austin, Texas.

${ }^{13}$ MIL-HDBK-5H, Change Notice 1, Battelle Memorial Institute, October, 2001.

${ }^{14}$ Knight, N.F. Jr, Rankin, C.C. and Brogan, F. A., "Controlling Progressive Failure Analyses using Artificial Viscous Damping," Proceedings of the AIAA/ASME/ASCE/AHS/ASC 42nd Structures, Structural Dynamics \& Materials Conference, April 16-19, Seattle, WA, AIAA paper No. 2001-14181, 2001.

${ }^{15}$ "HexWeb"M Honeycomb Attributes and Properties - A Comprehensive Guide to Standard Hexcel Honeycomb Materials, Configurations, and Mechanical Properties", Hexcel Composites, www.hexcel.com.

${ }^{16}$ ABAQUS User's Manual, Vol. 1-3, Version 6.4, Hibbitt, Karlsson, and Sorensen, Pawtucket, RI, 2003.

${ }^{17}$ Hysol ${ }^{\circledR}$ EA 9380, Epoxy Paste Adhesive Data Sheet, Henkel, www.aerospace.henkel.com 